\title{
The Design of Practice Training System Based on PLC Programmable Automatic Control
}

\author{
YE $\mathrm{Li}^{1, \mathrm{a}}$ \\ ${ }^{1}$ Guangxi Electric Polytechnic Institute, Nanning 530007, China \\ ayeligx@163.com
}

Keywords: Programmable Automatic Control; Practice Training System; PLC

\begin{abstract}
With the rise of modern industry and the rapid development of PLC, the requirement of industrial workers shift from high to high technology, gap technology talents, so that the vocational school talent training should pay attention to professional, technical and practical. As a condition of talents cultivation of hardware training equipment, can keep pace with The Times of replacement is particularly important. Based on PLC programmable automatic control system design and development, can promote technology talented person's raise produce certain. This system has played a core powerful programming of PLC control function, at the same time, the technology of sensor, electromagnetic, pneumatic, inverter consolidated, utmost ground close to the actual situation of the automatic production line, improve the simulation of this system.
\end{abstract}

\section{Introduction}

With the development of the microprocessor technology, the Programmable Logic Controller (PLC) is a combination of computer technology and automatic control technology developed a new kind of industrial Controller, through its internal storage procedures, to perform logical operations, sequential control, timing, counting and arithmetic operations such as user-oriented instructions, and through the digital or analog input/output control various types of machinery or production process, it will be the traditional relay Logic control technology, computer technology and communication technology, has a strong function, simple structure, convenient use, high reliability etc. [1]. After more than 30 years of development, PLC has been in metallurgy, machinery, electric power, transportation, weapon and other fields is widely used, greatly improve the automation level of the mechanical equipment and production lines [2].

As a condition of talents cultivation of hardware training equipment, and its upgrading of advancing with The Times is particularly important. Aimed at this situation, the school should adapt to social development needs, develop the corresponding professional and technical personnel, not only to let them understand and master the relevant professional knowledge, and have strong ability of problem analysis and solving ability. This system has played a core powerful programming of PLC control function, at the same time, the technology of sensor, electromagnetic, pneumatic, inverter consolidated, utmost ground close to the actual situation of the automatic production line, improve the simulation of this system.

\section{Programmable logic controller programming}

Main control logic part implemented jointly by controller and the programmable logic controller, the controller is responsible for receiving PC instruction and related parameters, and to achieve control of the programmable logic controller; Host controller programmable logic controller is responsible for the reception of the controller instructions and generate global clock signal and the signal, realize the infrared tubes launch logic and infrared receiving tube scan logic control [3-4]. The main control logic of the programmable logic control device of hardware implementation is shown in figure 1. Programmable logic control devices include the clock points frequency module, encode module, serial data transmission module. 


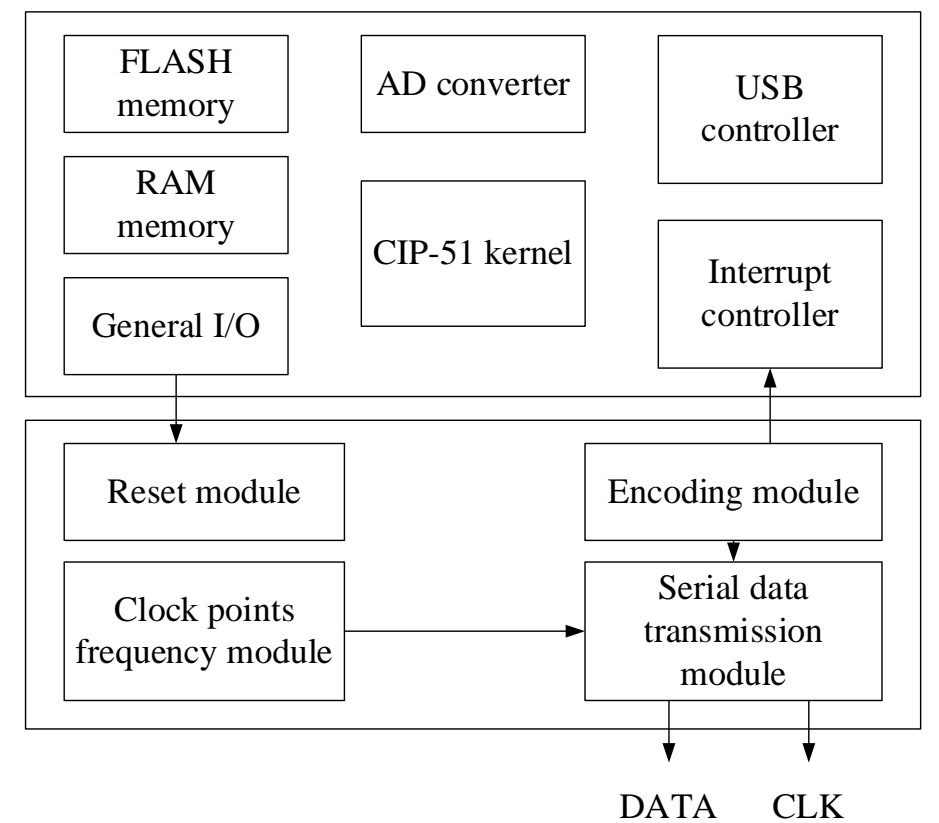

Figure 1. The logic circuit design of master controller

The clock points frequency module implements to produce a variety of synchronous clock signal. Touch screen system module using the same $40 \mathrm{MHZ}$ crystal active up to the clock signal source, crystal up the programmable logic control device in the main controller module clock input, the frequency division module generate the clock signal provided to other modules required for programmable logic control devices, thus the system clock is unified by the host controller programmable logic control devices provided frequency division, so that you can to ensure that the system of the various modules of signal synchronization.

The realization of decoding module is responsible for the overall control logic. The realization of the system of the whole control logic is performed by a similar counter function module, the module began counting after receiving controller synchronized trigger signal, and sends the data to the serial data transmission module, the data count to the corresponding infrared transceiver tube stops, so can facilitate the implementation of the extension of infrared touch screen size.

Serial data transmission module in IZC agreement on the basis of concise change, as the system module between data transmission protocol, realizes the data signal and clock signal synchronous transmission, makes the touch screen system coordination. Because of the signal transmission distance is longer, while avoiding interference environment, the transmission of signals on the hardware to $\mathrm{R}$ - 485 differential signal.

\section{The analysis of programmable automatic control training system based on PLC}

Programmable controller experiment system based on PLC consists of the guide rail type training platform, a typical electromechanical integration equipment of mechanical parts, touch screen, PLC module unit module, frequency converter module units, button module units, power supply module, simulation training module, terminal line production equipment and a variety of sensors, etc. [5]. Based on PLC programmable automatic control system of the whole machine working process is shown in figure 2 .

Integral structure with open and disassembling type, training device used for mechanical parts assembly, according to the existing mechanical parts assembly production equipment, also can add other mechanical parts assembly production equipment, make the whole device can flexible assemble according to the requirements of teaching or competition has the simulation function of electromechanical integration equipment. Module USES the standard structure and draw-out type module frame, strong interchangeability; According to the principle of production function and integrate learning function to determine the module content, make the teaching or race easy to choose the required module. The system includes mechanical and electrical integration of 
professional learning involved in, such as motor drive, mechanical transmission, pneumatic control, touch screen, programmable controller, sensors, such as frequency control of motor speed technology, provides students with a typical comprehensive training environment, so that the students learned in the past the only branch of the professional and basic knowledge, here can get a comprehensive knowledge, comprehensive training and practical application. Input by the touch screen control command, according to the process of the PLC program execution in the box, the figure shows that the program according to the direction of the arrow sequentially, when conditional judgment, according to the different conditions, to perform different results.

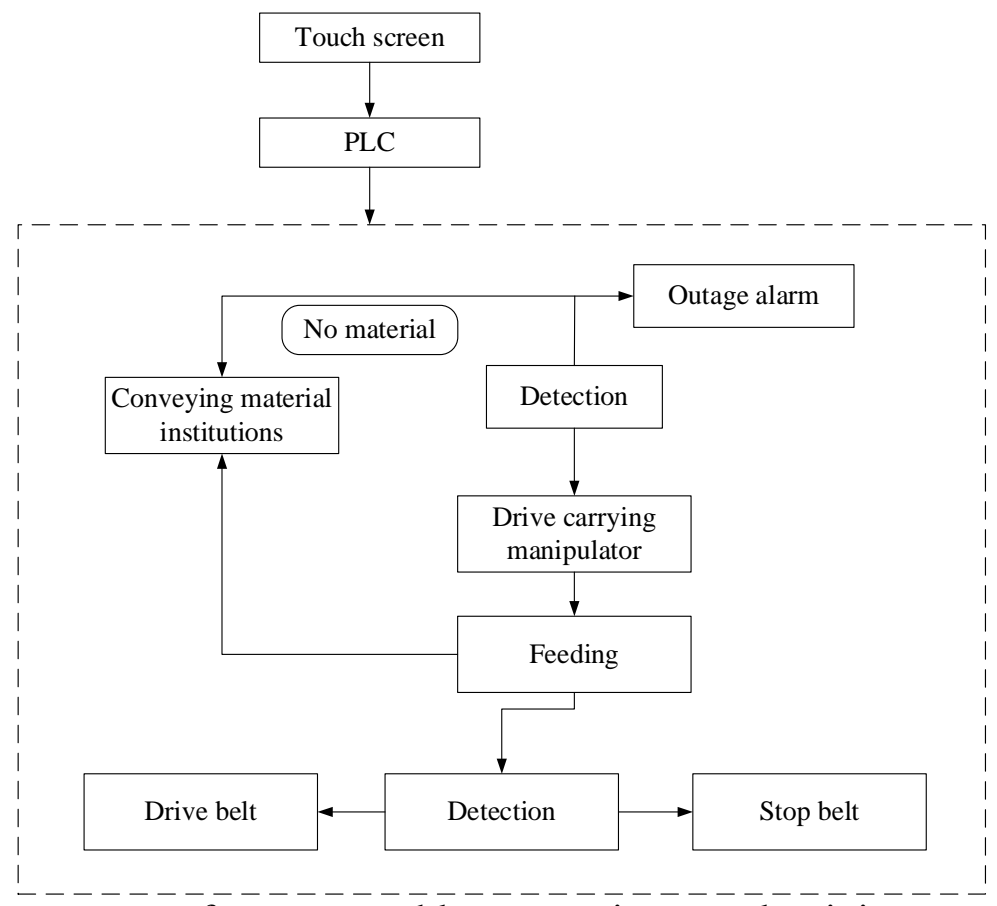

Figure 2. The working process of programmable automatic control training system based on PLC

\section{The practice test of the programmable automatic control training system}

Electromechanical integration equipment through the improvement and function development of the hardware has a certain improvement of its application. On programming, flexible use of sensor material recognition function, can according to the different requirements of engineering and the precision of the need to achieve, with three sensors and two sensor identification recognition, can also be based on material such as hardware improvement, more convenient identification of material types. Especially PLC series of n/n 3 BD board, can realize the computer and PLC, touch screen and PLC of online communication at the same time, each other and to reduce the PLC communication exchange use the mouth of the trouble, overcomes the defect of debugging the program cannot monitor at the same time. With a simple engineering design as an example to illustrate, according to the project purpose, draw a state diagram as shown in the 3.

Simulation on PLC programmable automatic control equipment of a workshop on material distribution, amount and type of materials on a touch screen Settings, conveyor belt allows moving in one direction only, when meet the workshop material sent to the corresponding position, the conveyor belt to stop, controlled by the cylinder push rod into its, when identifying material, touch screen should show the material information. First of all, the workshop materials needed to set by touch screen, and thus need to on the page object types and volume of material selection, including buttons and numerical input object, at the same time in the program should have corresponding Settings determine the object. Second conveyor allows moving in one direction only, this limits the three sensors were used to detect the thinking of the material, therefore can only use the photoelectric sensor and inductance sensor identification of materials or materials with the improved fast identification of materials, the project, with the former as the example to illustrate. Again, according to the material information, including material number and variety, need to use 
the transparency of artifacts, and superposition of three components, when the condition is met, according to otherwise hidden.

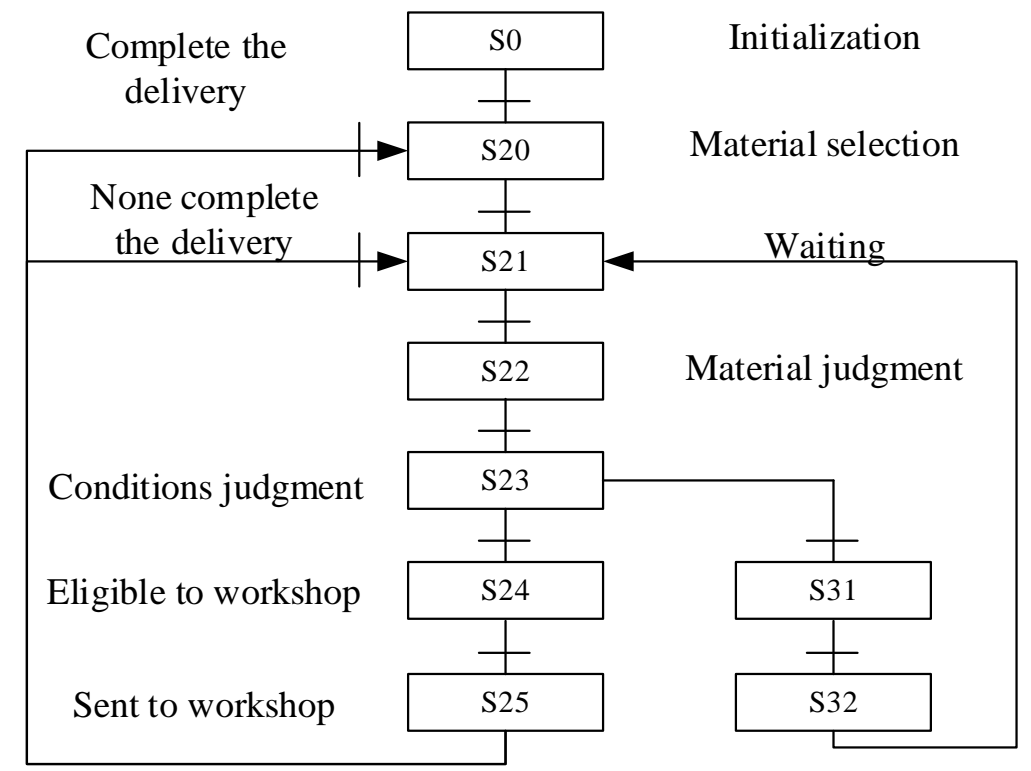

Figure 3. The flow chart of state

\section{Conclusion}

With the rise of modern industry and the rapid development of PLC, the requirement of industrial workers shift from high to high technology, gap technology talents, so that the school personnel training should pay attention to professional, technical and practical. In this paper, based on the mechanical and electrical integration of the laboratory equipment, PLC programmable controller, the PLC, frequency converter control, electrical control, pneumatic and function improvement research on the aspects such as man-machine interface, let the student practice and simulation of production, so as to effectively promote their brains, begin, to enhance their professional skills. Hope that through this research, and applies results into the skill teaching, really can achieve the above purpose, in order to improve the professional skill teaching effect.

\section{Acknowledgements}

This research is supported by scientific research project of guangxi education department (The construction of teaching resources sharing platform in configuration monitoring system, No. LX2014620)and scientific research project of Guangxi Electric Polytechnic Institute (Research of Industrial Configuration Software Application in Practice Teaching, No.2013KYY08) .

\section{Reference}

[1] R. Bayindir, Y. Cetinceviz: ISA transactions, Vol.50 (2011) No.2, p. 321.

[2] Z. Aydogmus, O. Aydogmus: Education, Vol.52 (2009) No.1, p. 126.

[3] M. Kano, M. Ogawa: Journal of Process Control, Vol.20 (2010) No.9, p.969.

[4] G. Valencia-Palomo, J.A. Rossiter: ISA transactions, Vol.50 (2011) No.1, p. 92.

[5] J.C.B. Gonzaga, L.A.C. Meleiro, and C. Kiang: Computers \& Chemical Engineering, Vol.33 (2009) No.1, p. 43. 